\title{
Sleep in Hospitalized Patients
}

\section{Anne Marie Morse, $\mathrm{DO}^{1}$ and Evin Bender, $\mathrm{MD}^{2}$}

\author{
Anne Marie Morse, $\mathrm{DO}^{1}$ \\ Assistant Professor, \\ Geisinger Commonwealth School of Medicine \\ Department of Child Neurology and Sleep Medicine \\ Geisinger Medical Center \\ Janet Weis Children's Hospital \\ 100 N. Academy Ave \\ Danville, PA 17820 \\ Email: amorse@geisinger.edu \\ Tel. 570.271.8254 \\ Fax 570.271.7923
}

\section{Evin Bender, MD $^{2}$}

Neurology Resident, PGY-3

Department of Neurology

Geisinger Medical Center

100 N. Academy Ave

Danville, PA 17820

Email: enbender@geisinger.edu 


\begin{abstract}
:
Hospitalized patients frequently have disordered and poor-quality sleep due to a variety of both intrinsic and extrinsic factors. These include frequent nighttime intrusions, insomnia related to pain and unfamiliar environments, dark conditions during the day with loss of natural light, and disruption of natural sleep cycle due to illness. Sleep wake disturbances can result in deleterious consequence on physical, emotional and cognitive status, which may impact patient satisfaction, clinical recovery, and hospital length of stay. Despite this, clinicians frequently fail to document sleep disturbances and are generally unaware of best practices to improve sleep quality in the hospital. The purpose of this review is to discuss sleep disturbances in hospitalized patients with a focus on causes of sleep disturbance, effect of poor quality sleep, high risk populations, considerations for surveillance and prevention, as well as pharmacologic and non-pharmacologic options for treatment.
\end{abstract}

Keywords: Hospitalized patients, sleep wake dysfunction, sleep disorders, circadian rhythm, sleep apnea 


\section{Introduction:}

Sleep is a homeostatic process that contributes to maintenance of health, cognition and mood stability. ${ }^{1,2}$ Normal sleep in an adult generally is about 7-9 hours per night and consists of $4-6,90-120$ minute cycles between non-rapid eye movement (REM) and REM sleep. ${ }^{3}$ Sleep wake dysfunction (SWD) refers to the disruption of the normal sleep wake relationship that can lead to the development of a transient or chronic sleep wake disorder (SD). This can be a result of reduced number of hours of sleep, poor quality sleep or inappropriate timing or stabilization between wake and sleep. When present, SWD can contribute to development and progression of co-morbid disease.

Approximately $10 \%$ of adults have a chronic SD that results in clinically significant symptoms ${ }^{4}$, with up to $50 \%$ of adults experiencing transient episodes of sleep wake dysfunction. Similarly, in pediatrics $20-30 \%$ of children are affected by a sleep disorder ${ }^{5}$ and up to $80 \%$ of children with chronic conditions have a co-morbid sleep disorder ${ }^{6-8}$. The risk of development of a chronic sleep wake disorder increases with age ${ }^{9}$. Patients with chronic medical disease are higher risk for experiencing co-morbid sleep wake disorders ${ }^{10}$. In fact, acute hospitalization for medical illness is a high-risk period for development or exacerbation of SWD, which can result in deleterious consequences ${ }^{11}$.

Hospitalized patients frequently report sleep quality while inpatient to be similar to the report of sleep quality of individuals who suffer from chronic SD. ${ }^{2}$ For instance, insufficient sleep, with an average of 5 hours of sleep per night, with multiple night time awakenings had been found in adult patients in a neurologic ward. ${ }^{1}$ There is a suggestion that the higher the acuity of illness the greater the impact on sleep. For example, pediatric patients in intensive care unit can experience up to a 54 percent reduction in sleep hours, while children in the general pediatric unit have been reported to have a 20-25 percent sleep loss. ${ }^{12}$ A closer examination of the causes of poor sleep, identification of high risk populations and the consequence of impaired sleep complemented with a review of surveillance 
techniques and management strategies for SWD may assist in improving early recognition and treatment of sleep disorders in hospitalized patients.

\section{Sleep Wake Dysfunction in Hospitalized Patients}

The most common acquired SD in hospitalized patients are insomnia and circadian rhythm disorders ${ }^{13-16}$. However, sleep disordered breathing (SDB), restless leg syndrome (RLS), and hypersomnia disorders can also be present and may be pre-existing or acquired, depending on medical comorbidity ${ }^{11,17}$. If there is pre-existing SD, this may become exacerbated by hospitalization and may predispose to development of additional SWD.

There are both intrinsic and extrinsic factors that contribute to development of SWD during hospitalization ${ }^{3,18-20}$. Intrinsic factors vary by patient, but can include primary medical illness, delirium, psychiatric co-morbidity (anxiety, depression, post-traumatic stress), pre-existing SD and physical pain or discomfort ${ }^{1,2,21,22}$. Pain and discomfort are generally the most frequently cited causes of SWD during hospitalization ${ }^{1}$.

Extrinsic factors that contribute to the development of SWD can be environmental noise, bright lights and irregular exposure to lighting, an unfamiliar environment with loss of normal bedtime routine, as well as repetitive clinical interventions, such as testing, clinical examinations and vitals, as well as medication administration ${ }^{23}$. Environmental noise can be due to equipment/alarms, medical staff, or hospital roommates. Roommates are commonly reported as the most disturbing factor for sleep ${ }^{1}$. A study evaluating acceptable environmental noise levels found that daytime noise of 59 decibel A (dBA) and nighttime noise of $50.5 \mathrm{dBA}$ did not result in SWD. ${ }^{24}$ However, median noise during the day was found to be $63 \mathrm{dBA}$ and $61 \mathrm{dBA}$ at night ${ }^{24}$. Noise levels in the ICU have been documented at an average peak of 150-200 dB with evening peaks $>80 \mathrm{~dB}$ between $12 \mathrm{AM}$ and $6 \mathrm{AM}$, contributing to about $30 \%$ of patient awakenings. ${ }^{25}$ 
Bright lighting and lighting irregularities may represent a more easily modifiable extrinsic factor for hospitalized patients. The strongest environmental cue for human circadian rhythm is the light dark cycle. ${ }^{26}$ Therefore, both irregular light exposure and bright light exposure during inappropriate circadian hours can result in circadian sleep dysfunction. Circadian misalignment has been demonstrated to be deleterious in multiple disease processes resulting in worsened disease severity, impaired treatment response and even decreased survivorship ${ }^{27}$.

\section{Effects of SWD in Hospitalized Patients}

Sleep wake disorders can result in deleterious consequence on physical, emotional and cognitive status, which may contribute to impaired recovery, prolonged length of stay, reduced subjective wellness and poor patient perception of hospitalized care $\mathrm{e}^{1,2,22,28}$. SWD and sleep deprivation have numerous deleterious effects (Figure 1), including autonomic dysfunction, impairment of the hypothalamic-pituitary-adrenal axis, and immunologic dysregulation ${ }^{29}$. Among hospitalized patients, reduced sleep duration and impaired sleep efficiency were independently associated with greater likelihood for impaired fasting glucose and hyperglycemia ${ }^{30}$. 
Figure 1: Consequences of Sleep Wake Dysfunction and Sleep Deprivation ${ }^{1,2,9,29-33}$

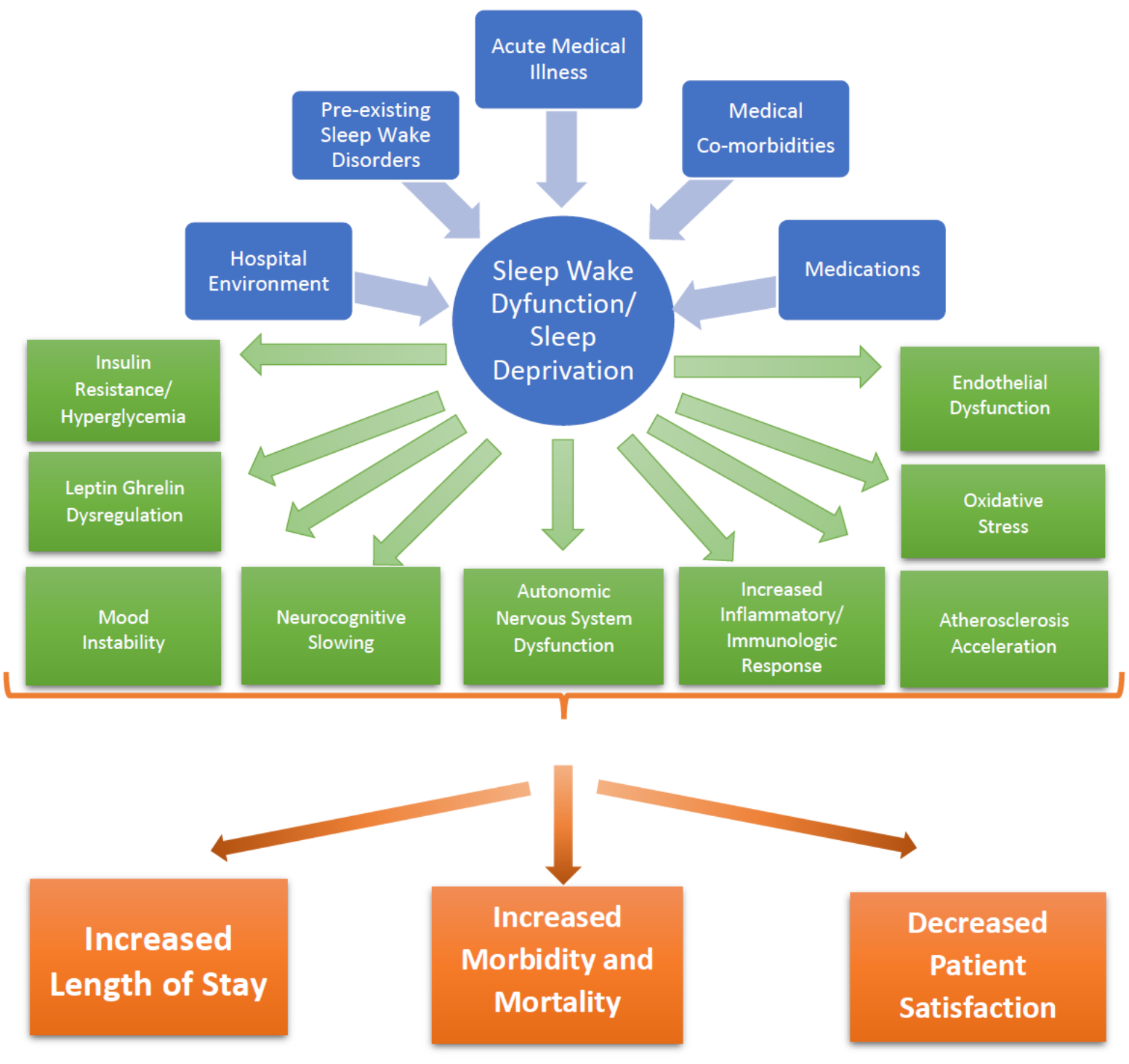


Poor quality sleep increases likelihood for development of delirium, anxiety and mood disorders ${ }^{34-36}$. Delirium and sleep deprivation have shared characteristics, including impaired attention, fluctuating mental status, disorganized thinking and altered level of consciousness ${ }^{35}$. Delirium occurs in up to $50 \%$ of hospitalized elderly patients, with strong associations in those greater than 80 and more common in men ${ }^{28,35}$. Delirium increases length of stay, likelihood for institutionalization post discharge sevenfold and risk for 6-month mortality when compared to those without delirium ${ }^{28}$.

The presence of pre-existing or newly developed SWD can significantly impact clinical outcomes. The most well studied co-morbid sleep disorder in hospitalized patients has probably been obstructive sleep apnea $(\mathrm{OSA})^{17,37-39}$. Surgical patients with OSA are more likely to experience postoperative hypoxemia and require ICU transfers with longer hospital stays ${ }^{17}$. Similarly, pregnant women with co-morbid OSA, have increased post-partum risk for maternal morbidity, cardiovascular morbidity, and in-hospital death ${ }^{37}$.

Circadian rhythm disturbances are gaining attention as a cause of SWD that negatively contributes to outcome measures in hospitalized patients ${ }^{15,16,32}$. The circadian rhythm does not only influence sleep wake patterns, but also dictates physiological and cellular functions that allows for adaption to cyclic environmental changes ${ }^{40}$. Evidence suggests the relationship between circadian function and disease is quite complex and multidirectional ${ }^{15,16,26,32,40-43}$. There is loss of normal diurnal variation in melatonin secretion in critically ill patients ${ }^{43}$, likely contributing to circadian misalignment. Patients hospitalized in the ICU have a loss of the normal rhythmic 24-hr physiologic profiles, including blood pressure, heart rate, body temperature, spontaneous motor activity, and the levels of melatonin and cortisol ${ }^{44}$. In addition, there is suggestion that there is a circadian effect on survival, which has been suggested to be related to circadian variability of immune responses ${ }^{42}$

High Risk Populations for SWD 
There are multiple factors that may influence the development of SWD during hospitalization (Table 1). Duration of slow wave sleep (SWS), total REM sleep and arousal thresholds decrease with age, culminating into reduced total sleep times with increased sleep fragmentation in individuals greater than 60 years old ${ }^{45,46}$. In addition, to the natural sleep changes that occur with age, advancing age is generally related to development of comorbid medical conditions. It is not uncommon for elderly patients to describe sleep complaints due to the presence or complications of these comorbidities ${ }^{47}$. 
Table 1: Risk Factors for SWD in Hospitalized Patients $9-11,15,16,30-32,45,48-54$ 


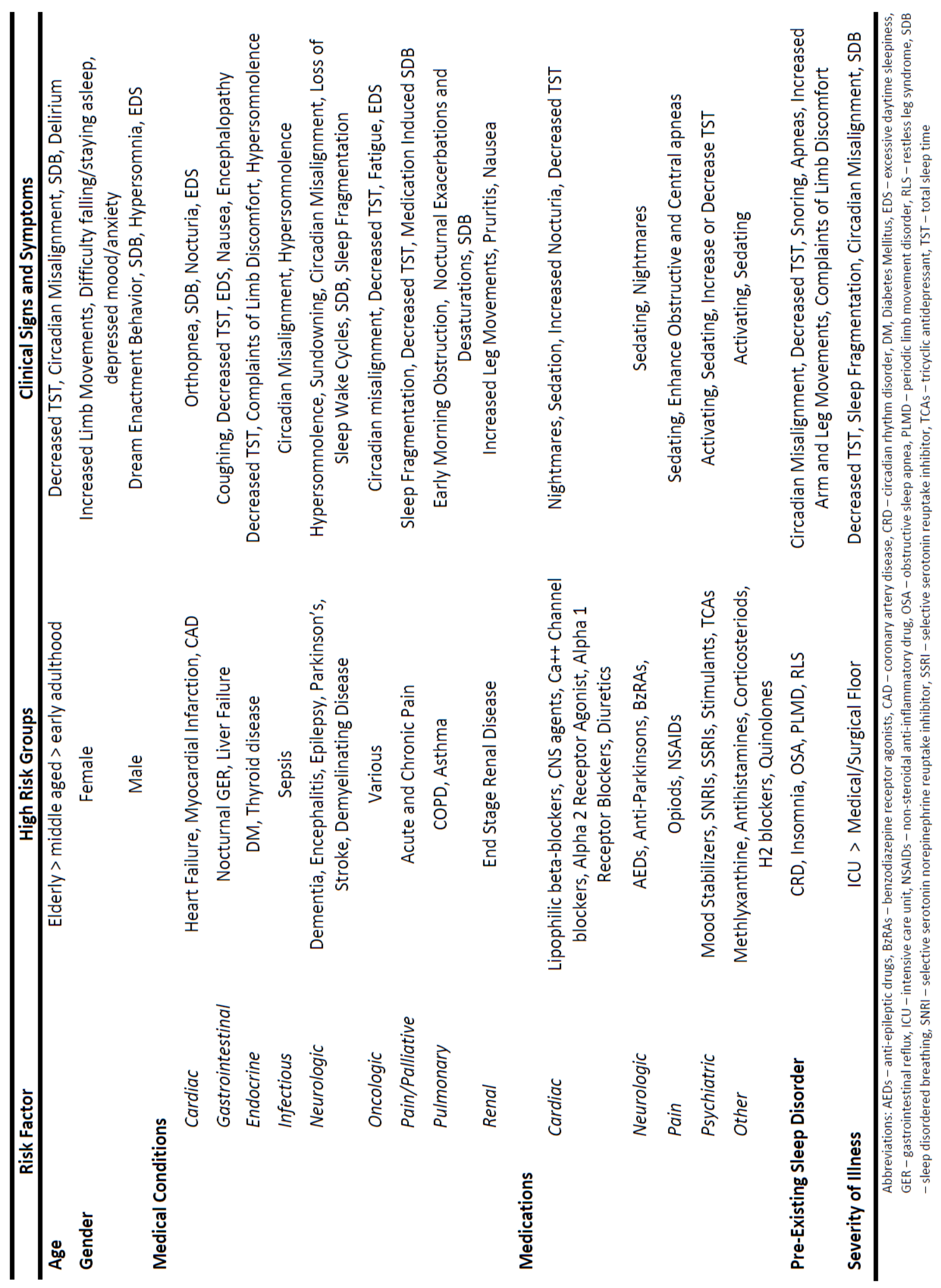


Comorbidities commonly associated with sleep disordered breathing and obstructive sleep apnea (OSA) include heart failure, coronary artery disease (CAD), and stroke, among others ${ }^{55-61}$. It is critical to identify OSA and sleep disordered breathing in these particular at risk populations because untreated OSA is an independent risk factor for initial and recurrent stroke, promotes CAD, and nearly triples the risk of mortality in patients with $C A D^{59-62}$. Diabetes is another condition associated with sleep changes, and it has been shown to correlate with lighter sleep with increased percent of time spent in stage 1 and 2 of sleep. ${ }^{45}$ COPD has also been shown to correlate with poor quality of sleep due to increased sleep latency, decreased total sleep time, and increased arousal. ${ }^{63}$ Parkinson's disease correlates with sleep fragmentation and REM behavior disorder (RBD), which can be particularly challenging to address in a hospital setting. ${ }^{64}$ Additionally, many conditions are associated with insomnia, the most extensively studied of which include cancer, untreated or poorly treated nocturnal pain, psychiatric conditions such as depression and anxiety, heart disease, and neurologic disease. ${ }^{23,47,54,65,66}$

Women have a slight predominance in occurrence of insomnia, as well as periodic limb movement disorder (PLMD) and restless leg syndrome (RLS). ${ }^{52,53}$ They are also statistically more likely to present to medical attention for sleep complaints and are therefore more likely to be prescribed hypnotic medications. ${ }^{53}$ Men on the other hand have a higher occurrence of OSA than women, and tend to have lighter sleep with greater time spent in stage 1 and stage 2 sleep compared to women. They also have a slight predominance for narcolepsy, and older men (age $>50$ ) have a slight predominance for REM behavior disorder compared to women. ${ }^{45,53}$ Sleep changes associated with older age include lighter sleep and decreased sleep efficiency with greater time spent in stage 1 and 2 sleep. ${ }^{45}$ Older age was also associated with greater occurrence of nocturnal myoclonus, RLS, and sleep disordered breathing. ${ }^{46}$ In addition to age, sex, and co-morbid conditions, many medications also are associated with SWD. Stimulant medications are associated with insomnia and circadian rhythm disorders. ${ }^{65}$ 
Benzodiazepines and opioids decrease the percent of time spent in later stages of sleep, and thereby reduce sleep efficiency. ${ }^{67,68}$ Antidepressants also reduce the time spent in REM sleep and increase REM onset latency, however this typically normalizes with long term treatment. An exception to this includes MAOIs which can cause REM sleep to be absent for months. ${ }^{69}$

\section{Physician Surveillance of SWD}

Despite the high prevalence of chronic sleep disorders in the general population and the knowledge that acute hospitalization is a high risk for development or exacerbation of a SWD, physicians frequently fail to address and document SWD in the hospital. One study performed on the general medical ward of a Veteran's Affairs hospital found that although $23 \%$ of patients admitted had a significant sleep symptom there was no medical record during their admission documenting sleep symptoms. ${ }^{70}$ Another study performed on a general medical floor at a non-profit academic hospital found that sleep histories were documented on only $9 \%$ of hospital charts, despite $68 \%$ of patients having conditions associated with obstructive sleep apnea (hypertension, obesity, stroke, and prior myocardial infarction)..$^{71}$

Standardized screening (Table 2) should be performed at admission to improve recognition of those with pre-existing sleep disorders and may help capture individuals who are higher risk for development of SWD during hospitalization. Re-evaluation during hospitalization may allow identification of those with secondary development of SWD and provide opportunity for intervention. Proactive methods to prevent development of SWD in hospitalized patients may include implementation of a sleep wake protocols that considers patient risk factors, hospital environment and timing of interventions coupled with reinforcement of regular sleep scheduling, sleep hygiene and pharmacologic interventions as needed $^{1,26}$. Research evaluating the efficacy and impact of these preventative strategies is limited. 
Table 2: Sleep Disorder Screening Tool ${ }^{72-76}$
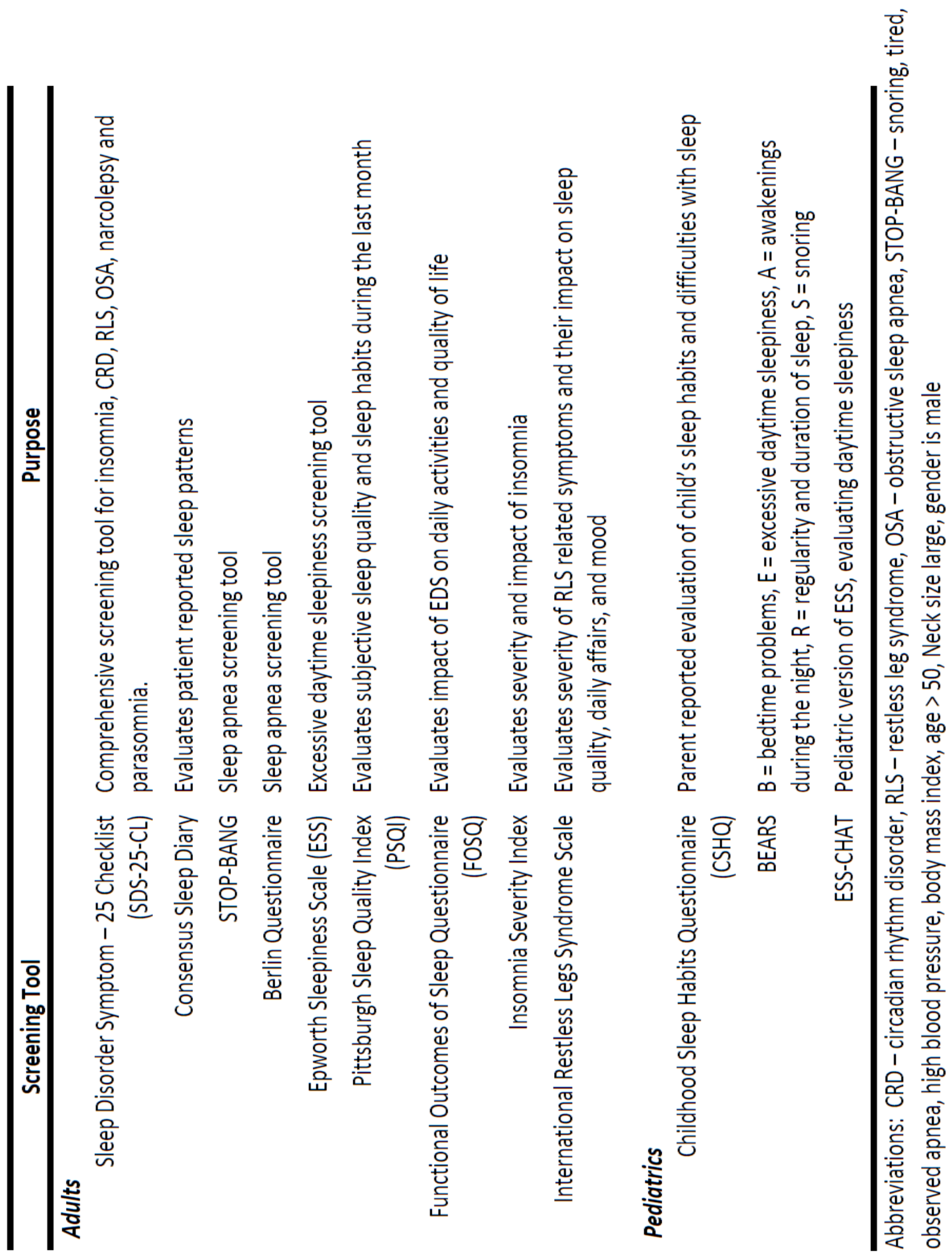


\section{Management of SWD in Hospitalized Patients}

\section{Non-Pharmacologic Interventions}

Non-pharmacologic interventions include strategic lighting, noise reduction techniques, and sleep rounds with sleep protocols featuring various implementations to aid with relaxation. A study on daytime light exposure and sleep found that exposure to at least 3000 Ix between the hours of 10:00 and 15:00 was likely to improve quality and duration of sleep in elderly patients. ${ }^{77}$ Supportive care directed at improved relaxation, such as use of warm blankets, warm milk, a white noise machine, hypoallergenic lotion, or a room spritzer, has been shown to improve perceptions of noise levels and reduce sleep latency in hospitalized patients. ${ }^{1}$ Use of three part sleep protocol, not only resulted in improved patient's self-reported sleep quality, but also significantly reduced use of sedative hypnotic drugs from $54 \%$ to $31 \% .^{21}$

While elderly and acutely ill patients are at risk for SWD while hospitalized, they are also more prone to serious adverse effects of sedative hypnotic drugs, including falls, delirium, and respiratory depression ${ }^{22}$. Additionally, sedative hypnotics have not consistently been proven to correlate with improved sleep quality. ${ }^{2,22}$ In many cases, it is therefore preferable to try non-pharmacologic interventions first line when treating SWD in these populations.

\section{Pharmacologic Interventions}

Sedative-hypnotic drug use is common, both in the general population and among hospitalized patients. Approximately $35 \%$ of patients are prescribed a home hypnotic for sleep ${ }^{2}$, and $45-70 \%$ of patients are prescribed a hypnotic during their hospitalization. ${ }^{22}$ Additionally, approximately $30 \%$ of patients are prescribed a new hypnotic in the hospital without any record of pre-admission use. ${ }^{2}$ The majority of SHDs used included benzodiazepines or zopiclone, but it is not uncommon for antidepressants, antipsychotics, antiemetics, and narcotics to be used for their sedating properties. ${ }^{2}$ 
Although the intention of sedative hypnotics is to improve sleep, use of these medications has not consistently proven to correlate with improved sleep quality, but instead has been associated with adverse cognitive outcomes and falls. ${ }^{2}$

\section{Discussion}

An improved understanding of the various factors that can influence sleep during hospitalization allows providers to better evaluate and treat sleep problems more systematically, but also creates an opportunity for systems to adapt preventative and surveillance practices. Sleep wake dysfunction is a multifactorial problem in hospitalized patients, resulting from a combination of the hospital environment, psychological stress, the patient's medical illness and treatments. Patients can be stratified by these features that may increase likelihood for development sleep difficulties during hospitalization (TABLE 1).

Implementing standardized screening for SD at admission and possibly during admission may help identify those with pre-existing sleep disorders and those who may have developed SWD during hospitalization. Treatment of SWD should be provided with both pharmacologic and non-pharmacologic options considered. Non-pharmacologic options are typically safer and have been shown to be beneficial. Proactive methods to prevent development of SWD in hospitalized patients may be considered. Further research is needed to evaluate efficacy in preventing SWD, as well as impact on length of stay, patient's perception, morbidity and mortality. 
Blibliography:

1. Thomas KP, Salas RE, Gamaldo C, et al. Sleep rounds: A multidisciplinary approach to optimize sleep quality and satisfaction in hospitalized patients. Journal of hospital medicine. 2012;7(6):508-512.

2. Frighetto L, Marra C, Bandali S, Wilbur K, Naumann T, Jewesson P. An assessment of quality of sleep and the use of drugs with sedating properties in hospitalized adult patients. Health and quality of life outcomes. 2004;2(1):17.

3. Knauert MP, Malik V, Kamdar BB. Sleep and sleep disordered breathing in hospitalized patients. . 2014;35(05):582-592.

4. Ram S, Seirawan H, Kumar SK, Clark GT. Prevalence and impact of sleep disorders and sleep habits in the united states. Sleep and Breathing. 2010;14(1):63-70.

5. Ivanenko A, Gururaj BR. Classification and epidemiology of sleep disorders. Child Adolesc Psychiatr Clin N Am. 2009;18(4):839-848. doi: 10.1016/j.chc.2009.04.005 [doi].

6. Richdale AL, Schreck KA. Sleep problems in autism spectrum disorders: Prevalence, nature, \& possible biopsychosocial aetiologies. Sleep medicine reviews. 2009;13(6):403-411.

7. Stores G, Wiggs L, Campling G. Sleep disorders and their relationship to psychological disturbance in children with epilepsy. Child Care Health Dev. 1998;24(1):5-19.

8. Hodge D, Carollo TM, Lewin M, Hoffman CD, Sweeney DP. Sleep patterns in children with and without autism spectrum disorders: Developmental comparisons. Res Dev Disabil. 2014;35(7):1631-1638. 
9. Wolkove N, Elkholy O, Baltzan M, Palayew M. Sleep and aging: 1. sleep disorders commonly found in older people. CMAJ. 2007;176(9):1299-1304. doi: 176/9/1299 [pii].

10. Ballard RD. Sleep and medical disorders. Prim Care. 2005;32(2):511-533. doi: S0095-4543(05)000291 [pii].

11. Young JS, Bourgeois JA, Hilty DM, Hardin KA. Sleep in hospitalized medical patients, part 1: Factors affecting sleep. Journal of hospital medicine: an official publication of the Society of Hospital Medicine. 2008;3(6):473-482.

12. Hinds PS, Hockenberry M, Rai SN, et al. Nocturnal awakenings, sleep environment interruptions, and fatigue in hospitalized children with cancer. . 2007;34(2).

13. Azad N, Byszewski A, Sarazin FF, McLean W, Koziarz P. Hospitalized patients' preference in the treatment of insomnia: Pharmacological versus non-pharmacological. Can J Clin Pharmacol. 2003;10(2):89-92.

14. Griffiths MF, Peerson A. Risk factors for chronic insomnia following hospitalization. J Adv Nurs. $2005 ; 49(3): 245-253$.

15. Knauert MP, Haspel JA, Pisani MA. Sleep loss and circadian rhythm disruption in the intensive care unit. Clin Chest Med. 2015;36(3):419-429.

16. Gazendam JA, Van Dongen HP, Grant DA, Freedman NS, Zwaveling JH, Schwab RJ. Altered circadian rhythmicity in patients in the ICU. Chest. 2013;144(2):483-489.

17. Kaw R, Pasupuleti V, Walker E, Ramaswamy A, Foldvary-Schafer N. Postoperative complications in patients with obstructive sleep apnea. Chest. 2012;141(2):436-441. 
18. Dobing S, Frolova N, McAlister F, Ringrose J. Sleep quality and factors influencing self-reported sleep duration and quality in the general internal medicine inpatient population. PloS one. 2016;11(6):e0156735.

19. Kokras N, Kouzoupis AV, Paparrigopoulos T, et al. Predicting insomnia in medical wards: The effect of anxiety, depression and admission diagnosis. Gen Hosp Psychiatry. 2011;33(1):78-81.

20. Missildine K, Bergstrom N, Meininger J, Richards K, Foreman MD. Sleep in hospitalized elders: A pilot study. Geriatr Nurs. 2010;31(4):263-271.

21. Ünsal A, Demir G. Evaluatıon of sleep quality and fatigue in hospitalized patients. Int J Caring Sci. 2012;5(3):311-319.

22. McDowell JA, Mion LC, Lydon TJ, Inouye SK. A nonpharmacologic sleep protocol for hospitalized older patients. J Am Geriatr Soc. 1998;46(6):700-705.

23. Raymond I, Nielsen TA, Lavigne G, Manzini C, Choinière M. Quality of sleep and its daily relationship to pain intensity in hospitalized adult burn patients. $P A I N^{\circledR} .2001 ; 92(3): 381-388$.

24. Park MJ, Yoo JH, Cho BW, Kim KT, Jeong WC, Ha M. Noise in hospital rooms and sleep disturbance in hospitalized medical patients. Environ Health Toxicol. 2014;29:e2014006. doi:

10.5620/eht.2014.29.e2014006 [doi].

25. Young JS, Bourgeois JA, Hilty DM, Hardin KA. Sleep in hospitalized medical patients, part 2: Behavioral and pharmacological management of sleep disturbances. Journal of Hospital Medicine: An Official Publication of the Society of Hospital Medicine. 2009;4(1):50-59. 
26. Czeisler CA, Kronauer RE, Allan JS, et al. Bright light induction of strong (type 0) resetting of the human circadian pacemaker. Science. 1989;244(4910):1328-1333.

27. Truong KK, Lam MT, Grandner MA, Sassoon CS, Malhotra A. Timing matters: Circadian rhythm in sepsis, obstructive lung disease, obstructive sleep apnea, and cancer. Annals of the American Thoracic Society. 2016;13(7):1144-1154.

28. Leukoff S, Evans D, Liptzin B. Delirium: The occurrence and persistence of symptoms among elderly hospitalised patients. Arch Intern Med. 1992;152(2):334-340.

29. Young JS, Bourgeois JA, Hilty DM, Hardin KA. Sleep in hospitalized medical patients, part 1: Factors affecting sleep. Journal of hospital medicine: an official publication of the Society of Hospital Medicine. 2008;3(6):473-482.

30. DePietro RH, Knutson KL, Spampinato L, et al. Association between inpatient sleep loss and hyperglycemia of hospitalization. Diabetes Care. 2017;40(2):188-193. doi: 10.2337/dc16-1683 [doi].

31. Smolensky MH, Portaluppi F, Manfredini R, et al. Diurnal and twenty-four hour patterning of human diseases: Acute and chronic common and uncommon medical conditions. Sleep medicine reviews. $2015 ; 21: 12-22$.

32. Truong KK, Lam MT, Grandner MA, Sassoon CS, Malhotra A. Timing matters: Circadian rhythm in sepsis, obstructive lung disease, obstructive sleep apnea, and cancer. Annals of the American Thoracic Society. 2016;13(7):1144-1154. 
33. Vgontzas AN, Chrousos GP. Sleep, the hypothalamic-pituitary-adrenal axis, and cytokines: Multiple interactions and disturbances in sleep disorders. Endocrinol Metab Clin North Am. 2002;31(1):15-36. doi: S0889-8529(01)00005-6 [pii].

34. Weinhouse GL, Schwab RJ, Watson PL, et al. Bench-to-bedside review: Delirium in ICU patientsimportance of sleep deprivation. Critical Care. 2009;13(6):234.

35. Watson PL, Ceriana P, Fanfulla F. Delirium: Is sleep important? Best Practice \& Research Clinical Anaesthesiology. 2012;26(3):355-366.

36. Sandberg O, Franklin KA, Bucht G, Gustafson Y. Sleep apnea, delirium, depressed mood, cognition, and ADL ability after stroke. J Am Geriatr Soc. 2001;49(4):391-397.

37. Louis JM, Mogos MF, Salemi JL, Redline S, Salihu HM. Obstructive sleep apnea and severe maternalinfant morbidity/mortality in the united states, 1998-2009. Sleep. 2014;37(5):843-849.

38. Bolona E, Hahn PY, Afessa B. Intensive care unit and hospital mortality in patients with obstructive sleep apnea. J Crit Care. 2015;30(1):178-180.

39. Khayat R, Jarjoura D, Porter $\mathrm{K}$, et al. Sleep disordered breathing and post-discharge mortality in patients with acute heart failure. Eur Heart J. 2015;36(23):1463-1469.

40. Kramer A, Merrow M. Circadian clocks. Springer; 2013.

41. Baskett J, Cockrem J, Todd M. Melatonin levels in hospitalized elderly patients: A comparison with community based volunteers. Age Ageing. 1991;20(6):430-434. 
42. Boivin DB, James FO, Wu A, Cho-Park PF, Xiong H, Sun ZS. Circadian clock genes oscillate in human peripheral blood mononuclear cells. Blood. 2003;102(12):4143-4145. doi: 10.1182/blood-2003-03-0779 [doi].

43. Mundigler G, Delle-Karth G, Koreny M, et al. Impaired circadian rhythm of melatonin secretion in sedated critically ill patients with severe sepsis. Crit Care Med. 2002;30(3):536-540.

44. Paul T, Lemmer B. Disturbance of circadian rhythms in analgosedated intensive care unit patients with and without craniocerebral injury. Chronobiol Int. 2007;24(1):45-61.

45. Redline S, Kirchner HL, Quan SF, Gottlieb DJ, Kapur V, Newman A. The effects of age, sex, ethnicity, and sleep-disordered breathing on sleep architecture. Arch Intern Med. 2004;164(4):406-418.

46. ROEHRS T, ZORICK F, SICKLESTEEL J, ROTH T, WITTIG R. Age-related Sleep-Wake disorders at a sleep disorder center. J Am Geriatr Soc. 1983;31(6):364-370.

47. Foley D, Ancoli-Israel S, Britz P, Walsh J. Sleep disturbances and chronic disease in older adults:

Results of the 2003 national sleep foundation sleep in america survey. J Psychosom Res. 2004;56(5):497502.

48. Mercadante S, Aielli F, Adile C, et al. Sleep disturbances in patients with advanced cancer in different palliative care settings. J Pain Symptom Manage. 2015;50(6):786-792.

49. Roscoe JA, Kaufman ME, Matteson-Rusby SE, et al. Cancer-related fatigue and sleep disorders. Oncologist. 2007;12(Supplement 1):35-42. 
50. Bitter T, Faber L, Hering D, Langer C, Horstkotte D, Oldenburg O. Sleep-disordered breathing in heart failure with normal left ventricular ejection fraction. European journal of heart failure. 2009;11(6):602608.

51. Dobing S, Frolova N, McAlister F, Ringrose J. Sleep quality and factors influencing self-reported sleep duration and quality in the general internal medicine inpatient population. PloS one. 2016;11(6):e0156735.

52. Ohayon MM, Roth T. Prevalence of restless legs syndrome and periodic limb movement disorder in the general population. J Psychosom Res. 2002;53(1):547-554.

53. Krishnan V, Collop NA. Gender differences in sleep disorders. Curr Opin Pulm Med. 2006;12(6):383389.

54. Ducloux D, Guisado H, Pautex S. Promoting sleep for hospitalized patients with advanced cancer with relaxation therapy: Experience of a randomized study. American Journal of Hospice and Palliative Medicine $^{\circledR} .2013 ; 30(6): 536-540$.

55. Peker Y, Kraiczi H, Hedner J, Loth S, Johansson A, Bende M. An independent association between obstructive sleep apnoea and coronary artery disease. Eur Respir J. 1999;14(1):179-184.

56. Bradley TD, Floras JS. Obstructive sleep apnoea and its cardiovascular consequences. The Lancet. 2009;373(9657):82-93.

57. Kasai T, Floras JS, Bradley TD. Sleep apnea and cardiovascular disease: A bidirectional relationship. Circulation. 2012;126(12):1495-1510. doi: 10.1161/CIRCULATIONAHA.111.070813 [doi]. 
58. Somers VK, White DP, Amin R, et al. Sleep apnea and cardiovascular disease: An american heart association/american college of cardiology foundation scientific statement from the american heart association council for high blood pressure research professional education committee, council on clinical cardiology, stroke council, and council on cardiovascular nursing in collaboration with the national heart, lung, and blood institute national center on sleep disorders research (national institutes of health). J Am Coll Cardiol. 2008;52(8):686-717.

59. Peker Y, Hedner J, Norum J, Kraiczi H, Carlson J. Increased incidence of cardiovascular disease in middle-aged men with obstructive sleep apnea: A 7-year follow-up. American journal of respiratory and critical care medicine. 2002;166(2):159-165.

60. Cherkassky T, Oksenberg A, Froom P, Ring H. Sleep-related breathing disorders and rehabilitation outcome of stroke patients: A prospective study. American journal of physical medicine \& rehabilitation. 2003;82(6):452-455

61. Dziewas R, Humpert M, Hopmann B, et al. Increased prevalence of sleep apnea in patients with recurring ischemic stroke compared with first stroke victims. J Neurol. 2005;252(11):1394-1398.

62. Marin JM, Carrizo SJ, Vicente E, Agusti AG. Long-term cardiovascular outcomes in men with obstructive sleep apnoea-hypopnoea with or without treatment with continuous positive airway pressure: An observational study. The Lancet. 2005;365(9464):1046-1053.

63. Cormick W, Olson LG, Hensley MJ, Saunders NA. Nocturnal hypoxaemia and quality of sleep in patients with chronic obstructive lung disease. Thorax. 1986;41(11):846-854.

64. Partinen M. Sleep disorder related to parkinson's disease. J Neurol. 1997;244(1):S3-S6. 
65. Corkum P, Moldofsky H, Hogg-Johnson S, Humphries T, Tannock R. Sleep problems in children with attention-deficit/hyperactivity disorder: Impact of subtype, comorbidity, and stimulant medication. J Am Acad Child Adolesc Psychiatry. 1999;38(10):1285-1293. doi: S0890-8567(09)63244-5 [pii].

66. Taylor DJ, Mallory LJ, Lichstein KL, Durrence HH, Riedel BW, Bush AJ. Comorbidity of chronic insomnia with medical problems. Sleep. 2007;30(2):213-218.

67. Murphy Jr GM, Kremer C, Rodrigues HE, Schatzberg AF. Pharmacogenetics of antidepressant medication intolerance. Am J Psychiatry. 2003;160(10):1830-1835.

68. Figueroa-Ramos MI, Arroyo-Novoa CM, Lee KA, Padilla G, Puntillo KA. Sleep and delirium in ICU patients: A review of mechanisms and manifestations. Intensive Care Med. 2009;35(5):781-795.

69. Wilson S, Argyropoulos S. Antidepressants and sleep. Drugs. 2005;65(7):927-947.

70. Meissner HH, Riemer A, Santiago SM, Stein M, Goldman MD, Williams AJ. Failure of physician documentation of sleep complaints in hospitalized patients. West J Med. 1998;169(3):146-149.

71. Namen AM, Landry SH, Case LD, McCall WV, Dunagan DP, Haponik EF. Sleep histories are seldom documented on a general medical service. South Med J. 2001;94(9):874-879.

72. J Klingman K, R Jungquist C, L Perlis M. Introducing the sleep disorders symptom checklist-25: A primary care friendly and comprehensive screener for sleep disorders. Sleep Medicine Research. $2017 ; 8(1): 17-25$.

73. Levenson JC, Troxel WM, Begley A, et al. A quantitative approach to distinguishing older adults with insomnia from good sleeper controls. Journal of Clinical Sleep Medicine. 2013;9(02):125-131. 
74. Luyster FS, Choi J, Yeh C, Imes CC, Johansson AE, Chasens ER. Screening and evaluation tools for sleep disorders in older adults. Applied Nursing Research. 2015;28(4):334-340.

75. Owens JA, Spirito A, McGuinn M. The children's sleep habits questionnaire (CSHQ): Psychometric properties of a survey instrument for school-aged children. SLEEP-NEW YORK-. 2000;23(8):1043-1052.

76. Owens JA, Dalzell V. Use of the 'BEARS'sleep screening tool in a pediatric residents' continuity clinic: A pilot study. Sleep Med. 2005;6(1):63-69.

77. Wakamura T, Tokura H. Influence of bright light during daytime on sleep parameters in hospitalized elderly patients. J Physiol Anthropol Appl Human Sci. 2001;20(6):345-351. 